\title{
Effective Irrationality Measures for Certain Algebraic Numbers
}

\author{
By David Easton
}

\begin{abstract}
A result of Chudnovsky concerning rational approximation to certain algebraic numbers is reworked to provide a quantitative result in which all constants are explicitly given. More particularly, Padé approximants to the function $(1-x)^{1 / 3}$ are employed to show, for certain integers $a$ and $b$, that

$$
\left|(a / b)^{1 / 3}-p / q\right|>c q^{-\kappa} \text { when } q>0 .
$$
\end{abstract}

Here, $c$ and $\kappa$ are given as functions of $a$ and $b$ only.

In 1964 Baker [1], improving a technique used by Siegel [8], was able to obtain effective irrationality measures for the function $(1-x)^{m / n}$ evaluated at certain rational points. In particular, he was able to show that for integers $p, q$ we have

$$
\left|2^{1 / 3}-p / q\right|>10^{-6} q^{-2.955} \text { when } q>0 \text {. }
$$

The technique was further refined by Chudnovsky [2] whose results, when applied to $2^{1 / 3}$, imply that for any $\varepsilon>0$ there exists a positive integer $q_{0}(\varepsilon)$ such that for integers $p, q$ we have

$$
\left|2^{1 / 3}-p / q\right|>q^{-(2.429+\varepsilon)} \quad \text { when } q>q_{0}(\varepsilon) .
$$

Chudnovsky's result is effective in the sense that it is possible in principle to work through the proof and compute, for any particular value of $\varepsilon$, a $q_{0}(\varepsilon)$ for which (2) holds. However, Chudnovsky does not undertake such computations.

In this article we rework Baker's proof using Chudnovsky's refinement, together with a Chebyshev-type result for primes in arithmetical progressions due to McCurley [6], and obtain the following quantitative result:

TheOREM. Let $a, b$ be integers with $0<b<a$. Define $d$ by

$$
d= \begin{cases}0 & \text { if } 3+(a-b), \\ 1 & \text { if } 3 \|(a-b), \\ 3 / 2 & \text { otherwise }\end{cases}
$$

Further, define $\lambda, \kappa, c$ and $q_{0}$ by

$$
\lambda=(.2328) 3^{d}\left(a^{1 / 2}-b^{1 / 2}\right)^{-2},
$$

Received May 9, 1985.

1980 Mathematics Subject Classification. Primary 10F25.

(C)1986 American Mathematical Society $0025-5718 / 86 \$ 1.00+\$ .25$ per page 


$$
\begin{array}{cc}
\text { (5) } & \kappa=1+\log \left(8.591(a+b) 3^{-d}\right)(\log \lambda)^{-1}, \\
\text { (6) } & c=1.69 \times 10^{-2}(a+b)^{-1}\left[.9302\left(a^{1 / 2}+b^{1 / 2}\right)^{-1}\left(a b^{2}\right)^{1 / 3}\left(a^{1 / 2}-b^{1 / 2}\right)\right]^{\kappa-1}, \\
\text { (7) } & q_{0}=\lambda^{300}\left[.9302\left(a^{1 / 2}+b^{1 / 2}\right)^{-1}\left(a b^{2}\right)^{1 / 3}\left(a^{1 / 2}-b^{1 / 2}\right)\right] .
\end{array}
$$

Then, assuming $\lambda>1$, we have for integers $p, q$

$$
\left|(b / a)^{1 / 3}-p / q\right|>c q^{-\kappa} \text { when } q>q_{0} .
$$

We remark that the Theorem yields an improvement on Liouville's Theorem provided $\kappa<3$, which occurs when

$$
(158.5)\left(3^{-3 d}\right)(a+b)\left(a^{1 / 2}-b^{1 / 2}\right)^{4}<1 .
$$

As a consequence of the Theorem we are able to obtain

Corollary. For the values of $\alpha, \kappa, c$ and $q_{0}$ given by the following table, we have, for integers $p, q$ that

$\begin{array}{cccc} & |\alpha-p / q|>c q^{-\kappa} & \text { when } q>q_{0} . \\ \alpha & c & \kappa & q_{0} \\ 2^{1 / 3} & 2.2 \times 10^{-8} & 2.795 & 0 \\ 6^{1 / 3} & 1.03 \times 10^{-17} & 2.405 & 10^{1976} \\ 10^{1 / 3} & 7.81 \times 10^{-10} & 2.619 & 0 \\ 15^{1 / 3} & 4.5 \times 10^{-7} & 2.933 & 0 \\ 17^{1 / 3} & 2.51 \times 10^{-10} & 2.3391 & 0 \\ 19^{1 / 3} & 1.1 \times 10^{-8} & 2.473 & 0 \\ 20^{1 / 3} & 3.84 \times 10^{-10} & 2.333 & 0 \\ 22^{1 / 3} & 5.16 \times 10^{-8} & 2.482 & 0 \\ 26^{1 / 3} & 7.8 \times 10^{-7} & 2.9099 & 0 \\ 28^{1 / 3} & 7.59 \times 10^{-7} & 2.899 & 0 \\ 37^{1 / 3} & 1.31 \times 10^{-8} & 2.427 & 0 \\ 39^{1 / 3} & 1.46 \times 10^{-11} & 2.313 & 0 \\ 42^{1 / 3} & 2.12 \times 10^{-7} & 2.766 & 0 \\ 43^{1 / 3} & 1.94 \times 10^{-8} & 2.506 & 0\end{array}$

It should be emphasized that in our proof certain choices must be made, which essentially correspond to fixing a value for $\varepsilon$ in (2). Unfortunately, decreasing the size of $\varepsilon$, and hence of $\kappa$, causes the value of $q_{0}$, as given by (7), to increase. Moreover, since in some of the estimates we use, we employ bounds which are not sharp, we are not able, in our proof, to take $\varepsilon$ to be arbitrarily small. For example, the smallest value of $\kappa$ which our proof can be made to yield in the case of $2^{1 / 3}$ is $\kappa=2.4862 \ldots$; here $q_{0}=10^{9 \times 10^{5}}$ and $c=10^{-2}$. It was our aim in making the choices we did, to obtain as small a value for $\kappa$ as possible while keeping $q_{0}$ sufficiently small that it is practical, at least for most of the values of $\alpha$ given in the Corollary, to compute and employ continued fraction expansions to remove the restriction the Theorem places on the size of $q$. The continued fractions were computed at the University of Waterloo on a Honeywell DPS 8/49 using a program written in MAPLE. 
Lastly, we remark that while we have here restricted our attention to cubic irrationalities, our proof can easily be modified so that, by employing McCurley [5, Theorem 1.2], we are able to obtain results similar to our Theorem for any function of the form $(1-x)^{m / n}$, where $m$ and $n$ are coprime integers with $1 \leqslant m<n$, $n \geqslant 10$ and $n$ not "exceptional" as defined in [5].

I would like to thank Professor C. L. Stewart for his encouragement and guidance in the preparation of this article.

Preliminary Results. The hypergeometric function ${ }_{2} F_{1}(a, b ; c ; z)$ is defined by ${ }_{2} F_{1}(a, b ; c ; z)=1+\sum_{n=1}^{\infty} \frac{a(a+1) \cdots(a+n-1) b(b+1) \cdots(b+n-1) z^{n}}{c(c+1) \cdots(c+n-1) n !}$.

When $c$ and $a$ are negative integers with $c<a$, the coefficients of $z^{n}$ for $n>|a|$ are understood to be zero. For $r$ a positive integer we define $X_{r}(z), Y_{r}(z)$ and $R_{r}(z)$ by

$$
\begin{aligned}
X_{r}(z) & =\frac{(r+1) \cdots(2 r)}{(2 / 3)(5 / 3) \cdots(r-1 / 3)}{ }_{2} F_{1}(-r,-r-1 / 3 ;-2 r ; 1-z) \\
Y_{r}(z) & =z^{r} X_{r}\left(z^{-1}\right) \\
R_{r}(z) & =\frac{(1 / 3)(4 / 3) \cdots(r+1 / 3)}{(r+1)(r+2) \cdots(2 r+1)}{ }_{2} F_{1}(r+2 / 3, r+1 ; 2 r+2 ; 1-z) .
\end{aligned}
$$

We shall employ the following Lemmas:

LEMMA 1. Let $r$ be a positive integer. Then for any real number $z$ with $0<z<1$,

$$
z^{1 / 3} X_{r}(z)-Y_{r}(z)=(z-1)^{2 r+1} R_{r}(z)
$$

Proof. We obtain (12) from (4.2) of [2] upon noting that with $\nu=1 / 3$, (9) agrees with $X_{r}(z)$ in (4.4) of [2], (10) agrees with $Y_{r}(z)$ in (4.1) of [2] and (11) agrees with (4.3) of [2].

LEMMA 2. Let $r$ be a positive integer, and define $\Delta_{r}$ to be the smallest positive integer such that $\Delta_{r} X_{r}(z)$ is a polynomial with integer coefficients. Then $3+\Delta_{r}$.

Further, let $a, b$ be integers with $0<b<a$, and suppose $d$ is as defined in (3). Define $d_{0}$ by

$$
d_{0}= \begin{cases}3 / 2+\log r / \log 3 & \text { if } d=3 / 2 \\ 0 & \text { otherwise. }\end{cases}
$$

Then $\Delta_{r} a^{r} 3^{d_{0}-d r} X_{r}(b / a)$ and $\Delta_{r} a^{r} 3^{d_{0}-d r} Y_{r}(b / a)$ are integers.

Proof. From (4.1) of [2], with $\nu=1 / 3$ we have

$$
X_{r}(z)=\sum_{l=0}^{r}\left(\begin{array}{l}
r \\
l
\end{array}\right) \frac{(3 r+1)(3 r+4) \cdots(3(r-l+1)+1)}{2 \cdot 5 \cdots(3 l-1)} z^{r-l}
$$

hence $3+\Delta_{r}$.

Our proof of the second half of Lemma 2 is based on the proof of Proposition 5.1 of [2].

We first note that if $3+(a-b)$, the result follows from the definition of $\Delta_{r}$ together with the observation that $X_{r}(z)$ and $Y_{r}(z)$ are both polynomials in $z$ of degree $r$. 
If $d \geqslant 1$, we write $a-b=3^{h} g$, where $\operatorname{gcd}(3, g)=1$. It follows from (9) that

$$
\begin{aligned}
\Delta_{r} X_{r}(b / a)= & \frac{\Delta_{r} \cdot r !}{(2 / 3)(5 / 3) \cdots(r-1 / 3)}\left(\begin{array}{c}
2 r \\
r
\end{array}\right) \\
& \times{ }_{2} F_{1}\left(-r,-r-1 / 3 ;-2 r ; 3^{h} g / a\right) \\
= & \frac{\Delta_{r} 3^{r} r !}{2 \cdot 5 \cdots(3 r-1)} \\
& \times \sum_{i=0}^{r}\left(\begin{array}{c}
2 r-i \\
r
\end{array}\right)\left(\prod_{k=r-i+1}^{r}(3 k+1)\right)(i !)^{-1}(-g / a)^{i} 3^{(h-1) i} .
\end{aligned}
$$

If $d=1$, we observe that $h=1$ and that

$$
r ! \sum_{i=0}^{r}\left(\begin{array}{c}
2 r-i \\
r
\end{array}\right)\left(\prod_{k=r-i+1}^{r}(3 k+1)\right)(i !)^{-1} x^{i}
$$

is a polynomial of degree $r$ with integer coefficients. Hence, since $3+\Delta_{r}$, $3^{-r} a^{r} \Delta_{r} X_{r}(b / a)$ is an integer.

If $d=3 / 2$, we note that $h \geqslant 2$ and apply Lemma 4.1 of [2] with $n=3, s=1$ and see that

$$
\sum_{i=0}^{r}\left(\begin{array}{c}
2 r-i \\
r
\end{array}\right)\left(\prod_{k=r-i+1}^{r}(3 k+1)\right)(i !)^{-1} 3^{[i / 2]} x^{i}
$$

is a polynomial of degree $r$ with integer coefficients. Hence the sum on the right side of (14) is a polynomial in $(-g / a)$ with integer coefficients. Thus, since $3+\Delta_{r}$, and since the exponent to which 3 divides $r$ ! is given by

$$
[r / 3]+[r / 9]+[r / 27]+\cdots \geqslant \frac{r}{2}-\left(\frac{\log r}{\log 3}+\frac{3}{2}\right),
$$

we see that $3^{d_{0}-d r} a^{r} \Delta_{r} X_{r}(b / a)$ is an integer.

We conclude the proof by noting that the above argument shows that $b^{r} \Delta_{r} 3^{d_{0}-d r} X_{r}(a / b)$ is an integer, and hence that

$$
a^{r} \Delta_{r} 3^{d_{0}-d r} Y_{r}(b / a)=\Delta_{r} 3^{d_{0}-d r} a^{r}\left(\frac{b}{a}\right)^{r} X_{r}(a / b)
$$

is an integer.

Lemma 3. Suppose $r$ is a positive integer. Then

$$
\Delta_{r} \mid \frac{2 \cdot 5 \cdots(3 r-1)}{r !} 3^{[r / 2]} \text {. }
$$

Further, if $\Delta_{r, l}$ denotes the contribution to $\Delta_{r}$ of all primes $p>(3 r)^{1 / 2}$, then

$$
\Delta_{r, l}<\exp \left\{r \sum_{A \geqslant 0} \sum^{\prime} \log p\right\},
$$

where the inner sum is taken over all primes $p \equiv 2 \bmod 3$ satisfying

$$
r /(A+1 / 3) \geqslant p>r /(A+2 / 3) \text {. }
$$

Proof. We verify (15) by noting that from Lemma 4.1 of [2] with $n=3, a=1$, $s=-1,2 \cdot 5 \cdots(3 r-1) 3^{[r / 2]}(r !)^{-1}$ is an integer, and moreover, from Lemma 4.2 of [2] with $n=3, s=1,2 \cdot 5 \cdots(3 r-1) 3^{[r / 2]}(r !)^{-1} X_{r}(z)$ is a polynomial with integer coefficients. 
To verify (16) we turn to Theorem 4.3 of [2]. In the proof of this theorem, Chudnovsky considers $\Delta_{r}^{(2)}$, the contribution to $\Delta_{r}$ of all primes $p>3 r^{1 / 2}$. Putting $n=3$, and $s=1$ in [2], we see that if $p \mid \Delta_{r}^{(2)}$ we must have $p \equiv 2 \bmod 3$, as is clear from the remarks made following (4.22). Moreover, the remarks made just prior to (4.20) show that $p^{2}+\Delta_{r}^{(2)}$; and from (4.22) we see that for some integer $A$, we must have $r /(A+1 / 3) \geqslant p>r /(A+2 / 3)$. This suffices to show (16) with $\Delta_{r}^{(2)}$ in place of $\Delta_{r, l}$. Our result follows upon observing that Chudnovsky's arguments are not affected by considering primes in the extended range $p>(3 r)^{1 / 2}$.

LEMMA 4. Let $r$ be a positive integer. If $\pi(r)$ denotes the number of primes less than $r$, we have

$$
\pi(r)<(1.001) r(\log r)^{-1}
$$

Further, if we put $\theta(r, 3,2)=\sum_{p \equiv 2 \bmod 3 ; p \leqslant r} \log p$, we have

$$
(.4075) r<\theta(r, 3,2)<(.5094) r \text { for } r \geqslant 47
$$

and

$$
(.4539) r<\theta(r, 3,2)<(.5094) r \text { for } r \geqslant 233 \text {. }
$$

Proof. We obtain (17) from (5.1) of [7]. The right-hand inequalities of (18) and (19) follow from Theorem 5.1 of [6], while the left-hand inequalities follow from Theorem 5.3 of [6].

Lemma 5. Let $a, b$ and $r$ be positive integers with $0<b<a$. Then if $X_{r}(z), Y_{r}(z)$ and $R_{r}(z)$ are given by $(9),(10)$ and $(11)$, respectively,

$$
\begin{aligned}
& X_{r}(b / a) Y_{r+1}(b / a) \neq X_{r+1}(b / a) Y_{r}(b / a) \\
& R_{r}(b / a)= \frac{(1 / 3)(4 / 3) \cdots(r+1 / 3)}{r !} \\
& \times \int_{0}^{1} t^{r}(1-t)^{r}(1-t(a-b) / a)^{-r-2 / 3} d t
\end{aligned}
$$

Proof. The proof of (20) is standard; see for instance the proof of (16) in [1]. We obtain (21) from (11) and (1.6.6) of [9].

Technical Lemmas. In this section we establish several estimates which we shall employ in the proof of the Theorem.

LEMMA 6. Let $r$ be an integer with $r \geqslant 300$. Then

$$
\Delta_{r}<\exp \{(1.4266) r\} \text {. }
$$

Proof. The proof is divided into two parts. First, we estimate the contribution to $\Delta_{r}$ of those primes $p \leqslant(3 r)^{1 / 2}$. We then estimate the contribution of those primes $p>(3 r)^{1 / 2}$.

To obtain the first estimate, we begin by recalling from Lemma 2 that $3+\Delta_{r}$.

We now proceed as Chudnovsky does in obtaining his upper bound for $\Delta_{r}^{(1)}$ in the proof of Theorem 4.3 of [2]. First, we note that from (15), if $p \leqslant(3 r)^{1 / 2}, p$ can contribute to $\Delta_{r}$ at most

$$
p^{[\log 3 r / \log p]} \leqslant 3 r
$$


Hence, if we denote the contribution to $\Delta_{r}$ of those primes $p \leqslant(3 r)^{1 / 2}$ by $\Delta_{r, s}$, we have

$$
\Delta_{r, s} \leqslant(3 r)^{\pi\left((3 r)^{1 / 2}\right)}
$$

Thus, from (17),

$$
\Delta_{r, s}<\exp \left\{2.002(3 r)^{1 / 2}\right\}
$$

and since $r \geqslant 300$, we have

$$
\Delta_{r, s}<\exp \{.2002 r\} .
$$

Denote, as in Lemma 3, the contribution to $\Delta_{r}$ of all primes $p>(3 r)^{1 / 2}$ by $\Delta_{r, l}$. We have from (16) that

$$
\begin{aligned}
& \Delta_{r, l}<\exp \left\{\sum_{A=0}^{\infty} \theta(r /(A+1 / 3), 3,2)-\theta(r /(A+2 / 3), 3,2)\right\} \\
&<\exp \left\{\sum_{A=0}^{5}(\theta(r /(A+1 / 3), 3,2)-\theta(r /(A+2 / 3), 3,2))\right. \\
&+\theta(r /(6+1 / 3), 3,2)\} .
\end{aligned}
$$

Hence, since $3 r / 2>233$, we have from (18) and (19) that

$$
\begin{aligned}
\Delta_{r, l} \leqslant & \exp \left\{(.5094)\left(\sum_{A=0}^{6} 3 r /(3 A+1)\right)\right. \\
& \left.\quad-(.4539)(3 r / 2)-(.4075) \sum_{A=1}^{5} 3 r /(3 A+2)\right\} \\
< & \exp \{(1.2264) r\} .
\end{aligned}
$$

Finally, from (23) and (24),

$$
\Delta_{r}=\Delta_{r, s} \Delta_{r, l}<\exp \{(1.4266) r\} .
$$

LemMa 7. Let $a, b$ and $r$ be integers with $0<b<a$ and $r \geqslant 300$. Let $d$ be given by (3), and let $d_{0}$ and $\Delta_{r}$ be as defined in Lemma 2. Put

$$
q_{r}=\Delta_{r} a^{r} 3^{d_{0}-d r} X_{r}(b / a) ; \quad p_{r}=\Delta_{r} a^{r} 3^{d_{0}-d r} Y_{r}(b / a) .
$$

Then $p_{r}$ and $q_{r}$ are integers with

$$
0<q_{r}<3.434\left(8.591 \cdot 3^{-d}(a+b)\right)^{r} \text {. }
$$

Proof. From Lemma 2, $p_{r}$ and $q_{r}$ are both integers.

The proof we shall give of (26) is essentially the proof of Lemma 3 of [1]. We begin by noting that from (13) we have

$$
\begin{aligned}
a^{r} X_{r}(b / a) & =a^{r} \sum_{l=0}^{r}\left(\begin{array}{l}
r \\
l
\end{array}\right) \frac{(r+1 / 3) \cdots(r-l+4 / 3)}{(2 / 3)(5 / 3) \cdots(l-1 / 3)}\left(\frac{b}{a}\right)^{r-l} \\
= & \prod_{k=1}^{r}(k-1 / 3)^{-1} \sum_{l=0}^{r}\left(\begin{array}{l}
r \\
l
\end{array}\right) \prod_{k=r-l+1}^{r}(k+1 / 3) \prod_{k=l+1}^{r}(k-1 / 3)\left(a^{l} b^{r-l}\right) .
\end{aligned}
$$


This, together with (25), gives the left-hand inequality of (26). Using the estimates

$$
\begin{aligned}
\prod_{k=r-l+1}^{r} & (k+1 / 3) \prod_{k=l+1}^{r}(k-1 / 3) \\
& \leqslant \prod_{k=r-l+1}^{r}(k+1) \prod_{k=l+1}^{r} k=r !\left(\begin{array}{c}
r+1 \\
l
\end{array}\right) \leqslant r ! 2^{r+1},
\end{aligned}
$$

we have

$$
\begin{aligned}
a^{r} X_{r}(b / a) & \leqslant r !\left(\prod_{k=1}^{r}(k-1 / 3)\right)^{-1} 2^{r+1} \sum_{l=0}^{r}\left(\begin{array}{l}
r \\
l
\end{array}\right) a^{l} b^{r-l} \\
& \leqslant 2(r !)\left(\prod_{k=1}^{r}(k-1 / 3)\right)^{-1}(2(a+b))^{r}
\end{aligned}
$$

Now

$$
\begin{aligned}
r !\left(\prod_{k=1}^{r}(k-1 / 3)\right)^{-1} & =\frac{3}{2} \prod_{k=2}^{r} \frac{3 k}{3 k-1}=\frac{3}{2} \exp \left\{\sum_{k=2}^{r} \log \left(1+\frac{1}{3 k-1}\right)\right\} \\
& <\frac{3}{2} \exp \left\{\sum_{k=2}^{r} \frac{1}{3 k-1}\right\}<\frac{3}{2} \exp \left\{\int_{1}^{r} \frac{1}{3 x-1} d x\right\} \\
& =\frac{3}{2} \exp \left\{\frac{1}{3} \log (3 r-1)-\frac{1}{3} \log 2\right\}<1.717 r^{1 / 3}
\end{aligned}
$$

Since $r \geqslant 300$,

$$
r !\left(\prod_{k=1}^{r}(k-1 / 3)\right)^{-1}<1.717(1.0064)^{r} .
$$

Further, since $r \geqslant 300, d_{0}=3 / 2+\log r / \log 3<(.02231) r$ and

$$
3^{d_{0}-d r} \leqslant 3^{(.02231-d) r} .
$$

The result follows from (22), (27), (28) and (29).

LEMMA 8. Let $a, b$ and $r$ be integers with $0<b<a$ and $r \geqslant 300$. Then,

$$
0<\left|(b / a)^{1 / 3}-p_{r} / q_{r}\right|<\frac{(.4445)(a-b)}{\left(a b^{2}\right)^{1 / 3} q_{r}}\left\{\frac{4.296}{3^{d}}\left(a^{1 / 2}-b^{1 / 2}\right)^{2}\right\}^{r}
$$

and

$$
p_{r} q_{r+1} \neq p_{r+1} q_{r}
$$

Proof. It is clear that (31) follows from (25) and (20). To verify (30), we first substitute $z=b / a$ in (12). Since from (26) $q_{r} \neq 0$, we have from (12), (21) and (25) that

$$
\begin{aligned}
\left|(b / a)^{1 / 3}-p_{r} / q_{r}\right|= & \frac{\Delta_{r} a^{r} 3^{d_{0}-d r}}{q_{r}}\left(1-\frac{b}{a}\right)^{2 r+1} \frac{(1 / 3)(4 / 3) \cdots(r+1 / 3)}{r !} \\
& \times\left|\int_{0}^{1} t^{r}(1-t)^{r}\left(1-\frac{(a-b)}{a} t\right)^{-r-2 / 3} d t\right| .
\end{aligned}
$$


Now the left-hand inequality of (30) follows upon observing that the integrand on the right side of (32) is positive for $0<t<1$. To obtain the right-hand inequality of (30) we first note that $(1-t(a-b) / a)^{-2 / 3} \leqslant(a / b)^{2 / 3}$ if $0 \leqslant t \leqslant 1$. Moreover, the function $t(1-t)(1-t(a-b) / a)^{-1}$ obtains a maximum value of $a\left(a^{1 / 2}+b^{1 / 2}\right)^{-2}$ on the range $0 \leqslant t \leqslant 1$. Hence

$$
\left|\int_{0}^{1} t^{r}(1-t)^{r}(1-t(a-b) / a)^{-r-2 / 3} d t\right| \leqslant(a / b)^{2 / 3}\left(a\left(a^{1 / 2}+b^{1 / 2}\right)^{-2}\right)^{r} .
$$

Further, in the same way as we obtained (28), we find that

$$
\begin{aligned}
\frac{(1 / 3)(4 / 3) \cdots(r+1 / 3)}{r !} & =4 / 9 \prod_{k=2}^{r} \frac{k+1 / 3}{k} \\
& <4 / 9 \exp \left\{1 / 3 \int_{1}^{r} \frac{d x}{x}\right\}<4 / 9(1.0064)^{r} .
\end{aligned}
$$

This, together with (29), (32), (33), and (22), implies (30).

Proof of Theorem. Let $\lambda$ be given by (4) and let $p, q$ be integers with $q$ satisfying

$$
\lambda^{r} \leqslant \frac{(1.076)\left(a^{1 / 2}+b^{1 / 2}\right) q}{\left(a b^{2}\right)^{1 / 3}\left(a^{1 / 2}-b^{1 / 2}\right)}<\lambda^{r+1}
$$

for some integer $r \geqslant 300$. Choose $R=r$ or $r+1$ so that $p q_{R} \neq p_{R} q$, as is possible in light of (31). Further, note that from (5)

$$
\lambda^{\kappa-1}=(8.591) 3^{-d}(a+b) .
$$

This, together with (26) and the left-hand inequality of (35), yields

$$
\begin{aligned}
q_{R} & <3.434\left((8.591) 3^{-d}(a+b)\right)^{r+1} \leqslant 3.434 \lambda^{(\kappa-1)(r+1)} \\
& \leqslant 3.434\left(\frac{1.076\left(a^{1 / 2}+b^{1 / 2}\right) \lambda}{\left(a b^{2}\right)^{1 / 3}\left(a^{1 / 2}-b^{1 / 2}\right)}\right)^{\kappa-1} q^{\kappa-1} .
\end{aligned}
$$

From the right side of (35), together with (4) and (30), we have

$$
\begin{aligned}
0 & <\left|(b / a)^{1 / 3}-p_{R} / q_{R}\right|<\frac{(.4445)(a-b)}{\left(a b^{2}\right)^{1 / 3} q_{R} \lambda^{r}} \\
& <(.4131) \frac{(a-b) \lambda\left(a^{1 / 2}-b^{1 / 2}\right)}{\left(a^{1 / 2}+b^{1 / 2}\right) q q_{R}}<\frac{(.0962) 3^{d}}{q q_{R}} .
\end{aligned}
$$

Since $d \leqslant 3 / 2$, we have

$$
\left|(b / a)^{1 / 3}-p_{R} / q_{R}\right|<\frac{1}{2 q q_{R}} .
$$

From (37) and (38) we have

$$
\begin{aligned}
\left|(b / a)^{1 / 3}-p / q\right| & \geqslant\left|p / q-p_{R} / q_{R}\right|-\left|(b / a)^{1 / 3}-p_{R} / q_{R}\right| \\
& \geqslant \frac{1}{q q_{R}}-\frac{1}{2 q q_{R}}=\frac{1}{2 q q_{R}} \\
& \geqslant \frac{1}{q^{\kappa}}\left\{.1456\left(\frac{\left(a b^{2}\right)^{1 / 3}\left(a^{1 / 2}-b^{1 / 2}\right)}{1.076\left(a^{1 / 2}+b^{1 / 2}\right) \lambda}\right)^{\kappa-1}\right\} .
\end{aligned}
$$


Hence, from (36) and (6),

$$
\begin{aligned}
\left|(b / a)^{1 / 3}-p / q\right| & >\frac{1}{q^{\kappa}}\left\{1.69 \times 10^{-2}(a+b)^{-1}\left(\frac{\left(a b^{2}\right)^{1 / 3}\left(a^{1 / 2}-b^{1 / 2}\right)}{1.076\left(a^{1 / 2}+b^{1 / 2}\right)}\right)^{\kappa-1}\right\} \\
& =\frac{c}{q^{\kappa}} .
\end{aligned}
$$

Proof of Corollary. To prove the corollary for $\alpha=2^{1 / 3}$, we apply the Theorem with $a=128, b=125$ to rationals of the form $5 q /(4 p)$ to obtain

$$
\frac{5}{4}\left|2^{-1 / 3}-q / p\right|>\frac{3.4 \times 10^{-5}}{(4 p)^{2.795}} \text { when } 4 p>10^{478} \text {. }
$$

Since it suffices to consider $q / p$ in the range $1<p / q<1.3$, we have

$$
\left|2^{1 / 3}-p / q\right|>\frac{3.4 \times 10^{-7}}{q^{2.795}} \text { when } q>10^{478} \text {. }
$$

To remove the restriction on $q_{0}$, we utilize the first 2000 terms in the continued fraction expansion for $2^{1 / 3}$. We begin by supposing that $q_{i}$ is the denominator of the $i$ th convergent to $2^{1 / 3}$, and that for some integers $p, q$ with $q_{i} \leqslant q<q_{i+1}$,

$$
\left|2^{1 / 3}-p / q\right|<\frac{3.4 \times 10^{-7}}{q^{2.795}} \text {. }
$$

Now if $a_{i}$ is the $i$ th partial quotient, we have the following well-known identities (the first follows from Theorem 9.6 of [4]; for the second see Theorem 182 of [3]):

$$
\frac{1}{\left(a_{i+2}+2\right) q_{i+1}^{2}}<\left|2^{1 / 3}-\frac{p_{i+1}}{q_{i+1}}\right|
$$

and

$$
\left|q_{i+1} 2^{1 / 3}-p_{i+1}\right|<\left|q 2^{1 / 3}-p\right| .
$$

These, together with (40), imply

$$
\frac{1}{\left(a_{i+1}+2\right) q_{i+1}}<\left|q 2^{1 / 3}-p\right|<\frac{3.4 \times 10^{-7}}{q^{1.795}}<\frac{3.4 \times 10^{-7}}{q_{i}^{1.795}} .
$$

Hence,

$$
\frac{2.9 \times 10^{6}}{\left(a_{i+2}+2\right) q_{i+1}} q_{i}^{1.795}<1
$$

Employing the identity $q_{i+1}=a_{i+1} q_{i}+q_{i-1}$, we have

$$
\frac{q_{i}}{q_{i+1}}=\frac{q_{i}}{a_{i+1} q_{i}+q_{i-1}}>\frac{1}{\left(a_{i+1}+1\right)},
$$

and hence from (41),

$$
\frac{2.9 \times 10^{6} q_{i}{ }^{795}}{\left(a_{i+2}+2\right)\left(a_{i+1}+1\right)}<1 .
$$

This, together with the observations that $q_{i} \geqslant \prod_{j=0}^{i} a_{j}$ and $\prod_{j=0}^{2000} a_{j}>10^{478}$, enables us to readily verify that for all integers $p, q$

$$
\left|2^{1 / 3}-p / q\right|>\frac{3.4 \times 10^{-7}}{q^{2.795}} \text { when } 0<q \leqslant 10^{478} \text {. }
$$


Hence, from (39) and (42), we have Corollary 1 for $\alpha=2^{1 / 3}$.

The rest of the Corollary is proved in a similar manner. We conclude by listing, for each value of $\alpha$, the values for $a$ and $b$ with which we obtain the result. We also list the values obtained for $q_{0}$.

\begin{tabular}{crrc}
$\alpha$ & $a$ & \multicolumn{1}{c}{$b$} & $q_{0}$ \\
$6^{1 / 3}$ & $467^{3}$ & $6 \cdot 257^{3}$ & $10^{1976}$ \\
$10^{1 / 3}$ & $5 \cdot 13^{3}$ & $2^{2} \cdot 14^{3}$ & $10^{846}$ \\
$15^{1 / 3}$ & $5^{2}$ & $3 \cdot 2^{3}$ & $10^{408}$ \\
$17^{1 / 3}$ & $18^{3}$ & $17 \cdot 7^{3}$ & $10^{1117}$ \\
$19^{1 / 3}$ & $19 \cdot 3^{3}$ & $8^{3}$ & $10^{802}$ \\
$20^{1 / 3}$ & $20 \cdot 7^{3}$ & $19^{3}$ & $10^{1141}$ \\
$22^{1 / 3}$ & $11 \cdot 5^{3}$ & $2^{2} \cdot 7^{3}$ & $10^{789}$ \\
$26^{1 / 3}$ & $3^{3}$ & 26 & $10^{417}$ \\
$28^{1 / 3}$ & 28 & $3^{3}$ & $10^{422}$ \\
$37^{1 / 3}$ & $10^{3}$ & $37 \cdot 3^{3}$ & $10^{890}$ \\
$39^{1 / 3}$ & $39^{2} \cdot 2^{3}$ & $23^{3}$ & $10^{1216}$ \\
$42^{1 / 3}$ & $7^{2}$ & $6 \cdot 2^{3}$ & $10^{498}$ \\
$43^{1 / 3}$ & $43 \cdot 2^{3}$ & $7^{3}$ & $10^{751}$
\end{tabular}

The continued fraction expansions for the above values of $\alpha$ are available from the author upon request.

Department of Pure Mathematics

University of Waterloo

Waterloo, Ontario N2L 3G1, Canada

1. A. BAKER, "Rational approximation to $2^{1 / 3}$ and other algebraic numbers," Quart. J. Math. Oxford Ser. (2), v. 15, 1964, pp. 375-383.

2. G. V. ChudnovsKy, "On the method of Thue-Siegel," Ann. of Math., v. 117, 1983, pp. 325-382.

3. G. H. HARDY \& E. M. Wright, An Introduction to the Theory of Numbers, 4th Ed., Oxford Univ. Press, London, 1960.

4. W. J. LeVeque, Topics in Number Theory, Addison-Wesley, Reading, Mass., 1956.

5. K. S. MCCURLEY, "Explicit estimates for the error term in the prime number theorem for arithmetic progressions," Math. Comp., v. 42, 1984, pp. 265-286.

6. K. S. MCCuruey, "Explicit estimates for $\theta(x ; 3 ; l)$ and $\psi(x ; 3 ; l)$," Math. Comp., v. 42, 1984, pp. 287-296.

7. J. B. Rosser \& L. SChoenfeld, "Sharper bounds for the Chebyshev functions $\theta(x)$ and $\psi(x)$," Math. Comp., v. 29, 1975, pp. 243-269.

8. C. L. Siegel, "Die Gleichung $a x^{n}-b y^{n}=c$," Math. Ann., v. 114, 1937, pp. 57-68.

9. L. J. Slater, Generalized Hypergeometric Functions, Cambridge Univ. Press, London, 1966. 